\title{
Long-Term Expression with Cationic Polymer Derived from a Natural Polysaccharide:
}

\section{Schizophyllan}

Takeshi Nagasaki, Masaya Hojo, Atsushi Uno, Taku Satoh, Kazuya Koumoto, Masami Mizu, Kazuo Sakurai, Seiji Shinkai

Size exclusion chromatography. The size exclusion chromatography set up consisted of HPLC LC-10A from Shimaz, Kyoto, Japan and YMC-Pak Diol-120 column (8.0 mmID x 300mmL) from YMC Co. Ltd., Kyoto, Japan. Compounds were detected by fluorescent detector RF-10A from Shimaz. As an eluent, $0.25 \mathrm{M}$ acetate buffer was used. A flow rate of $0.5 \mathrm{~mL} / \mathrm{min}$ was applied. Compound concentration was $1 \mathrm{~g} / \mathrm{L}$ and $10 \mu \mathrm{L}$ were injected. Sample preparation was conducted as follows. 5(and-6-)-carboxy-X-rhodamine, succinimidyl ester (5(6)-Rox, SE) from Molecular Probes, Eugene, OR was added to a DMF solution of APPD41(34K) and N, N'-bis(3-aminopropyl)propanediamine to prepare rhodamine-labled Rox-APPD41(34K) and Rox-APPD, respectively . Mixtures were stirred at $30^{\circ} \mathrm{C}$ for 24 $\mathrm{h}$ in the dark. Rox-APPD41(34K) was purified by ultrafiltration using a filter with a cut-off of $10^{4} \mathrm{Da}$. Rox-APPD was purified using gel filtration LH-20 with methanol as an eluent. The relation between the molecular weight and the retention time was calibrated by using monodispersed poly(vinylpyridinium bromide) from Polymer Standard Service, Mainz, Germany. Determined molecular weight of RoxAPPD41(34K) are estimated as $4 \times 10^{4}$ Da which supports predicted structure of the product of APPD41(34K).

(a)

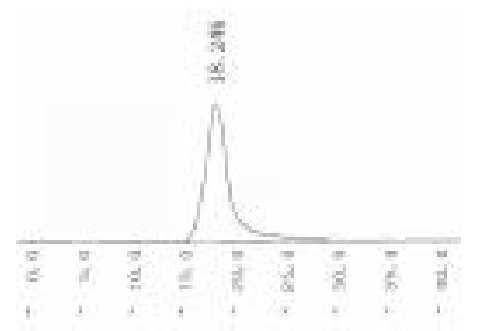

(b)

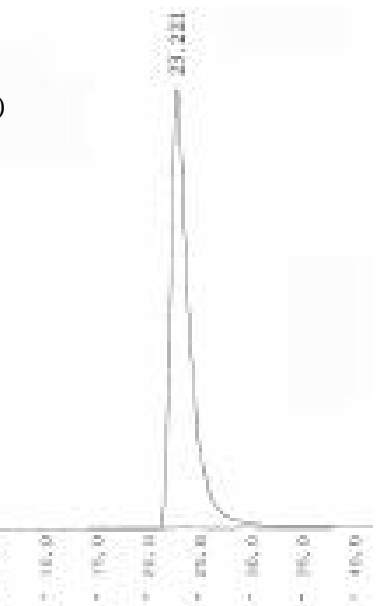

Figure 1. Size exclusion chromatograms of Rox-APPD41(34K) (a) and Rox-APPD (b). 
Transfection in the presence of endocytosis inhibitors. In order to estimate the uptake mechanism of cationic SPG/DNA complex, transfection was carried out in the presence of endocytosis inhibitors such as monensin, nigericin, chloroquine, and chlorpromazine. Before transfection with cationic polymers, COS-1 cells were preincubated for 30 min with either chlorpromazine $(28 \mu \mathrm{M})$, chloroquine $(200 \mu \mathrm{M})$, nigericin $(1 \mu \mathrm{M})$, and monensin $(10 \mu \mathrm{M})$ at $37^{\circ} \mathrm{C}$. Then, cells were washed once with serum free medium and applied for transfection assay. Moreover, we have examined cytotoxicity under experimental conditions in the presence of endocytosis inhibitors. Although we have performed in the manner similar to previously reported method by others. (for example: T. Niidome et al, Biomaterials, 21, 1811(2000)), In our case, all compounds showed high cytotoxicity under the conditions we have done. The results were represented in Figure 2 in supporting information. The decrease of transfection efficiencies should mainly be due to non-specific cytotoxiccity of used endocytosis inhibitors. Instead of use of the experiment with endocytosis inhibitor, therefore, we have confirmed the mechanism of cellular uptakes by another method.

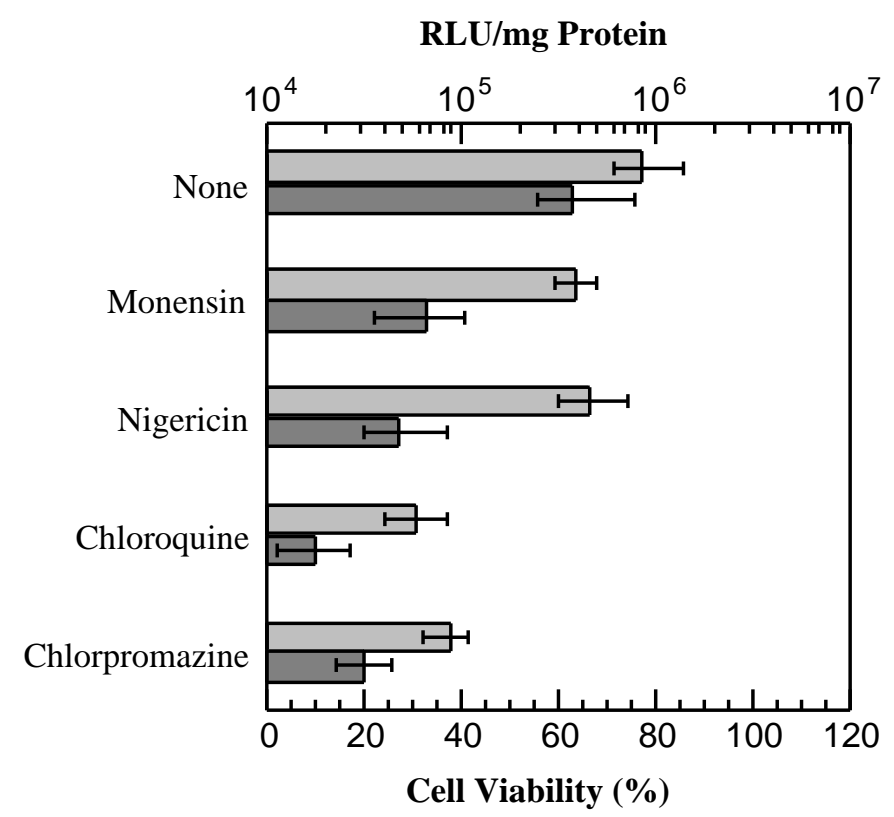

Figure 2. Effects of the endocytosis inhibitors on the transfection efficiency and cytotoxicity of APPD46(80K). Before incubation with the DNA complex, COS-1 cells were preincubated for $30 \mathrm{~min}$ with either monensin $(10 \mu \mathrm{M})$, nigericin $(1 \mu \mathrm{M})$, chloroquine $(200 \mu \mathrm{M})$, and chlorpromazine $(28 \mu \mathrm{M})$. Slashed bars indicate the relative transfection efficiency and dotted bars indicate the cell viability. Relative transfection efficiency is shown as relative light units (RLU)/mg of protein on the upper ordinate. Cell viability is shown as percentage of living cells on the lower ordinate. 